\title{
RoboMusicKids - Music Education with Robotic Building Blocks
}

\author{
Jacob Nielsen, Niels K. Bærendsen, Carsten Jessen \\ University of Southern Denmark \\ The Maersk Mc-Kinney Moller Institute \\ Centre for Playware \\ Campusvej 55, DK-5230 Odense M, Denmark \\ raider@mmmi.sdu.dk,mail@nielsk.dk, jessen@litcul.sdu.dk
}

\begin{abstract}
Being able to express oneself musically and experiment with music composition is traditionally determined by one's ability to play an actual instrument with a certain degree of craftsmanship. The lack of skills often makes it difficult to make children and young people experience the joy of musical creativity, which is an essential element in music education. This paper presents a pilot project where modular robotics is used to create a platform for creative musical expression that allows users to experiment with musical genres without any prior musical knowledge or skills.
\end{abstract}

\section{Introduction}

This RoboMusicKids project investigates how music education can be facilitated by the use of elements from the field of modular robotics. The technology used is intelligent blocks, I-BLOCKS, which are able to communicate with each other when physically connected and which are also able to detect the 3D structure that they are part of.

A lot of toys currently exist that can play sounds or music depending on the user's or the environment's interaction with them. Most of these have a preset library of sounds and/or tunes, which can be played back in only a few different ways - unless we are talking music instrument toys. These toys, therefore, do not allow the user to really experiment with composition and for instance try out different genres. It is our wish to create a platform for creative musical expression and experimentation, which does not require any pre-learned skills from the user.
Using the I-BLOCKS as a tool for musical experimentation, we allow children to play with professionally created musical pieces and thereby create their own mix and arrangement from a large number of overall possible variations. Our goal is that as the children play with variations of different genres, they thereby learn the characteristics of these, which is an important part of teaching musical comprehension. It is our hope that this hands-on musical experience also will sustain the children's interest in music creation and first and foremost keep them playing and learning.

The combination of modular robotics and music in RoboMusicKids is in our view a new approach to music teaching that allows children to learn by doing through manipulation with physical objects and gain knowledge about music in the same natural manner as young children commonly learn to know their environment. In relation to learning and technology in general, RoboMusicKids is an example of how learning can be transformed by altering the behaviour of the objects that we allow children to get their hands on.

The project is cross-disciplinary and combines two very different scientific areas, robotics and pedagogical research. In the following we will first introduce the IBLOCKS technology as a tool for experimenting with music, then describe results from user experiments, and close with a brief discussion of the possible advantages linked to the use of new music technology.

\section{I-BLOCKS Technology}

The I-BLOCKS (see Figure 1) are cubic modular robotic building blocks that can communicate with each other when connected. Each cube can communicate with up to 4 of its 6 possible neighbours and is fully self-contained with respect to power, connectors and processing. At the edges of the 4 
communicating sides of a cube are 4 RGB LEDs, which can light up in 4096 different colours. The IBLOCKS communicate locally via IR-transceivers, and can be internally expanded to support global wireless radio (XBEE) communication as well, in order to facilitate 'structure - structure' or 'structure - device' communication. Each I-BLOCK makes use of a 3D accelerometer to detect its orientation with respect to gravity. This makes it able to detect, for instance, which side is facing down.

The I-BLOCKS connect to each other using magnets, allowing for uni-sex connection at 90-degree angles. At the electronic centre of each I-BLOCK is the Atmel ATMEGA1280 8-bit microcontroller, which takes care of all processing including peripheral device communications etc. The I-BLOCKS hardware is encapsulated by black polyurethane (PUR) shell that has a soft rubber-like feel, with hard plastic plate lids in top and bottom in which charge plugs, programming connectors, sensors and actuators are integrated.

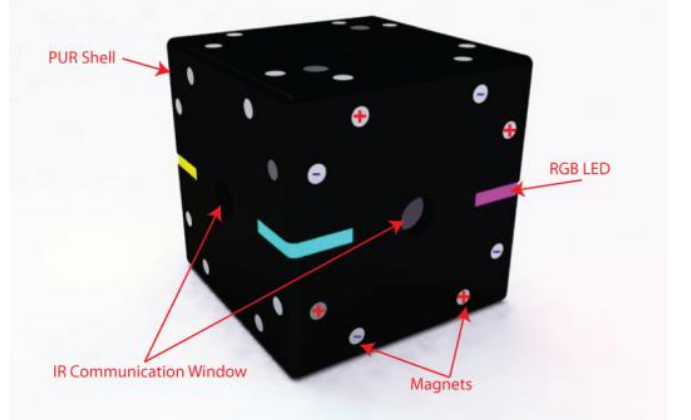

Figure 1. An explained visualization of the I-BLOCK.

An I-BLOCK is assembled as shown in Figure 2. The individual block can be expanded to support sensor, actuator and communication additions of which can be mentioned:

- Sensors: LDR, Flex, Stretch, Sonar, Microphone

- Actuators: LCD Text Display, LCD Graphic Display, Synth Sound, Vibrator

- Communication: USB, XBEE

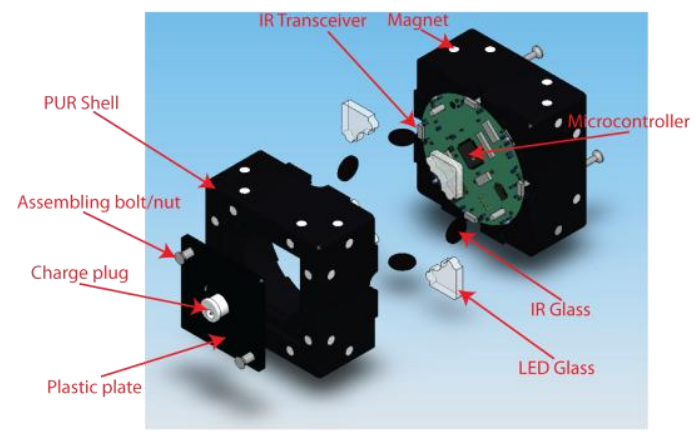

Figure 2. I-BLOCK explained assembly drawing.

The I-BLOCKS have been developed in several prototypes since year 2000, and are meant as a general platform for exploring physical programming - or "programming by building". The construction with IBLOCKS results not only in the development of a physical structure, but also in the development of a functionality of that physical structure. The functionality is a product of the sensor input, the actuator output, the communication and the processing of the individual I-BLOCKS.

There are many possible applications for this type of technology, and some of those explored so far [1][2] include arithmetic, language, neural networks, technology learning, programming, and with this project now also music composition.

We name our technology "modular robotic building blocks" because of our group's (www.adaptroncis.dk) strong relations to the fields of embodied AI (modular robotics, multi-agent systems etc.) and because of our definition on robotics: "A robot is defined as a programmable machine which autonomously performs a variety of tasks. A robot's behaviour distinguishes itself from a computer program by its interaction with the physical surroundings via sensors and actuators."

Our modules do not contain moving actuators, but both the light and sound output are actuators and by using these as feedback to a user's handling of the IBLOCKS they do invite the user to act as a "interactuator" to the system.

As written above, the I-BLOCKS should be considered a truly distributed system, and even though we use a PC as a generator of the music in the project mentioned here, this PC should just be considered a sound actuator I-BLOCK because it is interfaced in the same way as the other I-BLOCKS. The user does not even need to know about the existence of the PC, all that is needed is the audible feedback. 


\section{Existing Music-making Technologies and Concepts}

There exists numerous music-making technologies on the market today, and a few of these will be mentioned briefly in this section.

If we start on the pure software side, we have products such as Garageband from Apple [3]. This product is extremely user friendly and allows the user to compose music pieces from a large library of loops as well as standard audio and midi recording. The loops are presented to the user in a specific browser and can be dragged and dropped into the current project - even while the music is playing. The new version of Garageband includes a feature named "Magic Garageband" which allows the user to select from different genres of music (jazz, rock, funk, pop etc.) and then a pre-composed piece of music is played for the given genre. The different instruments for a given genre is presented to the user as "icons" on a virtual stage and can be selected to be modified to other variations of that instrument - while the music is playing. For instance the bass instrument in a jazz number can be changed from "walking" to "fretless" to "lounge" to "funky" etc. In this way, the user can setup a suitable accompaniment to her own performance (singing, playing etc.) The functionalities of "Magic Garageband" resemble very much what will be presented in this article, although this is true only from a functional point of view. Other software products that ought to be mentioned is Ableton Live [4], which we also use in this project, and Propellerhed Reason [5], which shares some of the functionalities of Garageband as well as also being very user friendly.

\subsection{Technological Products and Concepts}

Gil Weinberg, the director of the music technology programme at Georgia Tech, made the Beatbugs [6], which are hand-held percussive instruments that allow the creation, manipulation and sharing of rhythmic motifs through a simple interface. The Beatbugs (Figure 3) can be connected in a network, thereby letting the players form large-scale collaborative compositions.

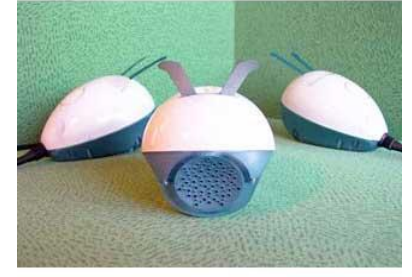

Figure 3. Beatbugs.

Sony Block Jam [7] is a musical interface controlled by the arrangement of 25 tangible blocks. By arranging the blocks (Figure 4) musical phrases and sequences are created, allowing multiple users to play and collaborate. The system takes advantage of both graphical and tangible user interfaces.

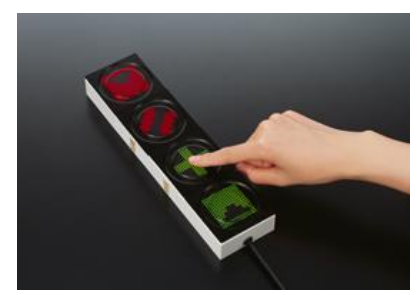

Figure 4. Sony Block Jam.

Percussa AudioCubes [8] as shown in Figure 5 is a tangible user interface allowing sound designers and music trainers to intuitively explore and create dynamically changing sounds. A new sound is created manipulating cubes, changing their orientation and distance.

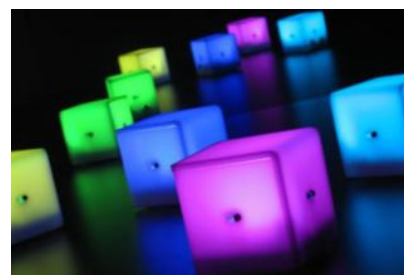

Figure 5. Audiocubes.

Besides the technologies mentioned above, a concept named RoboMusic [9] has been developed by Henrik Hautop Lund and Martin Ottesen. In RoboMusic, a number of robotic devices are used as instruments and the tunes are composed as a behaviourbased system. The RoboMusic concept supports the things presented in this article on the behavioural level although in another scenario.

\section{Musical Scenario}

We wanted to create a scenario where children can experiment freely with music composition, using a set 
of already composed pieces in different musical genres. As mentioned in the introduction our goal was not to teach children to make their own music from scratch, but instead to teach them the characteristics of different genres as, for instance, jazz genres, rock genres etc. which is an important part of teaching musical comprehension.

Using the specifications described above as a starting point we have tried to design an interaction scenario, where the user identifies the functionality and behaviour of the I-BLOCKS through musical feedback. So it has been a goal for us to make the different musical pieces distinguishable in order to make the user truly perceive the system.

The music created by the user is computed and played back on a PC, using the Ableton Live (C) music software as a playback unit responding to midi messages coming from the I-BLOCKS. In order to allow the blocks to "talk midi" to a PC we have made a wireless device, named "Midi Box" that converts serial wireless data coming from a XBEE-enabled I-BLOCK into midi signals. By using wireless technology we allow users to manipulate the blocks freely just like conventional building blocks.

The pieces of music that has been made for this project have all been constructed using these rules: There are six predefined instruments (varying according to genre) and within each piece of music there are up to six variations per instrument, and there can be an unspecified number of different instruments. When the user grabs an I-BLOCK, representing an instrument, the block's orientation - which side is facing down - determines the variation of that specific instrument. The I-BLOCK LEDs change colour depending on their orientation, in order to make it possible for the user to remember and activate specific variations. Each of the instrument I-BLOCKS has been given a colour to represent a specific instrument. For instance, the colour coding for a rock number has been implemented as follows:

- Red: Drums

- Blue: Bass

- Green: Keyboard

- Yellow: Guitar

- Cyan: Melody

- Pink: Human (Vox)

The colours are all easily distinguishable from a user's point of view.

The musical setup can be seen from Figure 6. Note the black XBEE-enabled I-BLOCK, which communicates wirelessly with the Midi Box, and therefore has to be present in every construction, in order for the PC to generate any music.

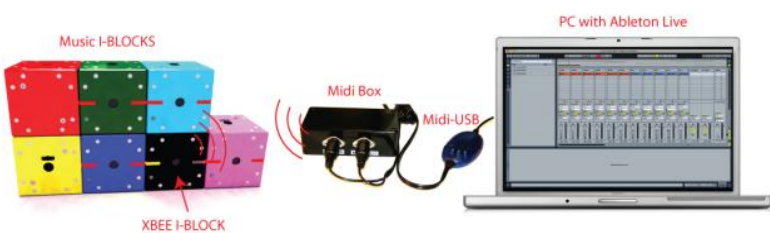

Figure 6. Music setup with I-BLOCKS, Midibox and PC.

The music style of the pieces created so far includes rock, funk, jazz, reggae, rockabilly, surf-rock, pop and hip-hop of the 80s. The music is mainly loop-based, meaning that when active, each variation of each instrument is playing a certain time and then repeating itself over and over until it is finally deactivated when the user removes the current instrument I-BLOCK from the structure or shifts its orientation.

A short example: A user connects a yellow guitar IBLOCK to the black XBEE-enabled I-BLOCK. Immediately the PC-music software starts playing one of six guitar tracks depending on how the block is rotated when connected. Another user might then add a red drum I-BLOCK to the structure, which will additionally start a drum track in the music. More instruments can be added, and others removed, and if the user chooses to rotate the entire structure, all the instruments present will start playing a different variation.

This manipulation with musical pieces is a kind of "sampling in real time" which is known from music software on computers (e.g. Ableton Live and Garage Band). The difference between such software and RoboMusicKids is, of course, the physical buildingblocks that allow for a more intuitive hands-on approach.

\section{Scenario Extensions}

In order to expand the ways the users can interact with the music to change the general sound of the compositions and also as a means to possibly involve more users in the composition process, we have come up with two extensions to the general scenario described above. Both of these come in the form of XBEE-enabled I-BLOCKS

The first extension is a guitar-solo instrument IBLOCK. The user interacts with this block by shaking it and thereby activating solo-tracks in the music. The harder the block is shaken, the more "intense" the sound of the chosen guitar-solo track, increasing in 
both note speed and tonal range. The level of shaking is also indicated on the I-BLOCK LEDs, which are fading from green to red depending on the shaking rate. We make use of the built-in accelerometer to detect the shaking of the block.

This kind of shaking interaction is meant to physically activate the user, making her activity level directly readable from the music as well as giving her the impression of actually being part of the music and thereby enhance the user's comprehension. This is particular the case in genres like rock and jazz, where solos and improvisation often are significant characteristics.

The second extension is named the effect I-BLOCK, and as its name might indicate, it can be used to turn up and down specified effects anywhere in the music. The effect I-BLOCK is currently influencing the melody part of the music, turning up and down effects such as cut-off frequency, harmony, resonance and portamento, depending on which melody variation is currently playing. Using the effect I-BLOCK, the user has the possibility to alter the music in more or less audible ways, again making her interaction directly influencing the music.

\section{User Experiments}

During the development of the music integrated in the I-BLOCKS we ran a number of tests and experiments in order to figure out how users responded to the music as well as the way in which the blocks enabled them to navigate this. The following section focuses on two experiments carried out in an afterschool centre and presents some of the observations we made in the execution of these.

\subsection{After-school experiments}

We carried out two user experiments at an afterschool centre (see Figure 7) which were of an explorative nature, as our focus was towards discovering ways to improve both the music implemented in the I-BLOCKS and ways to experiment with this as well as discovering the projects' potential regarding musical comprehension, learning and creativity. The participating children, aged between 10 and 12, were divided into groups of four. Some of these groups consisted of only boys or girls and some were mixed groups. Some of the children had prior experience with formal instrumental music tuition while others had little or no experience. All group sessions were recorded on video.

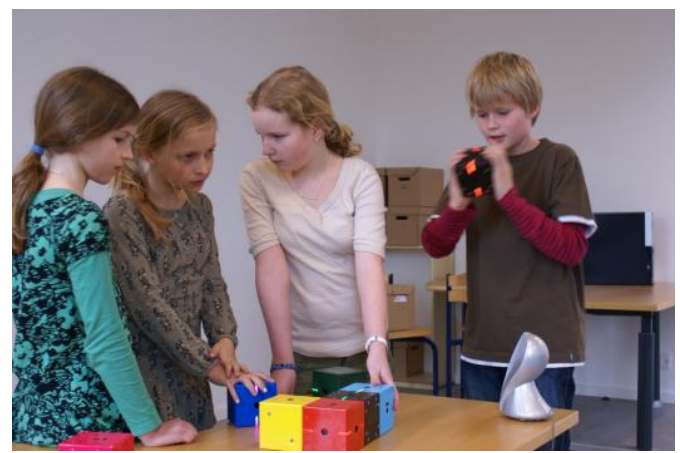

Figure 7. After-school experiments in Birkerød

The following is a description of some of the main findings as observed in the participants' use of the IBLOCKS. In this description an overall pattern regarding the structure of events observed in each session is presented, serving also as a possible outline of the learning process among the participants.

\subsection{Description of the sessions and their overall structure}

Reviewing the recorded sessions, we were able to roughly divide each group session into three steps or phases. As hinted above, the mentioning of these steps serves as a description of the actual use of the IBLOCKS as well as a framework for the later mentioning of perspectives in regard to music-making and musical experimentation with new technology. The three steps observed in the experimental sessions are as follows:

1. Exploratory use. Together as a group the children explored different ways of connecting the I-BLOCKS and the different musical segments and variations. This step was characterized by the children's fascination of the technology itself and the fact that by combining the I-BLOCKS they were able to initiate music. Everyone in the group would at this point actively connect and disconnect blocks and turn the structure around in an exploratory manner. No particular interest in the qualitative musical output and the actual combination of instruments and musical loops was apparent. The main interest among the children at this point was to activate and "checking out" the different loops, and - to a certain degree - find out which colours represented which instrument or sound.

As in many other regards, exploration of something - for instance a toy that one is not familiar with - is a natural approach. Therefore the explorative way in which the participating children approached this new toy was to be expected. What we saw was children employing the basic learning principles, which leading 
education theoreticians like Piaget, Dewey, and Vygotsky have described as, for instance, "active learning", "hands-on learning" and "experimental learning", and which they have pointed to as fundamental for children's learning. [10]

Of particular interest is the observation of cooperation as well as turn-taking among the participants during their further exploration and musicmaking, as described in the following.

2. Collaborative music-making. Following the exploratory phase, the children started to pay more attention to the actual musical output of their collaborative use of the I-BLOCKS. Typically this involved discussion among the participants regarding which instruments were audible during their construction of a structure and which variation of this particular instrument they preferred. Typically one participant would start off by connecting an I-BLOCK as a starting point, choosing a loop that they were particularly fond of. Another participant would connect another block, choosing a loop which they felt accompanied the first loop. From here on the group would experiment with the different loops and the structure of the blocks. At times this involved turning the entire structure around and thereby changing the entire musical output, and at other times turning single blocks, changing only the musical output of this particular instrument. The music-making and the learning approach in this phase were still very much a collaborative effort, but did, however, at this point involve a key element of turn-taking allowing the participants to single-handedly control the blocks and the position of the structure, having the other participants suggesting changes and supplying ideas.

3. Individual music-making. In each session we encouraged the participants to create their own musical piece or "mix" (Figure 8), having complete control over the blocks. At this stage it was particularly clear that I-BLOCKS served as a useful tool for musical expression, composition and performance. To a varying degree the participants would examine each block to choose the instrument variation they wanted to use and be very selective in this process. Often they would leave one or two blocks out of the mix in order to receive the output or sound they wanted, avoiding the overall mix getting clustered or "muddy".

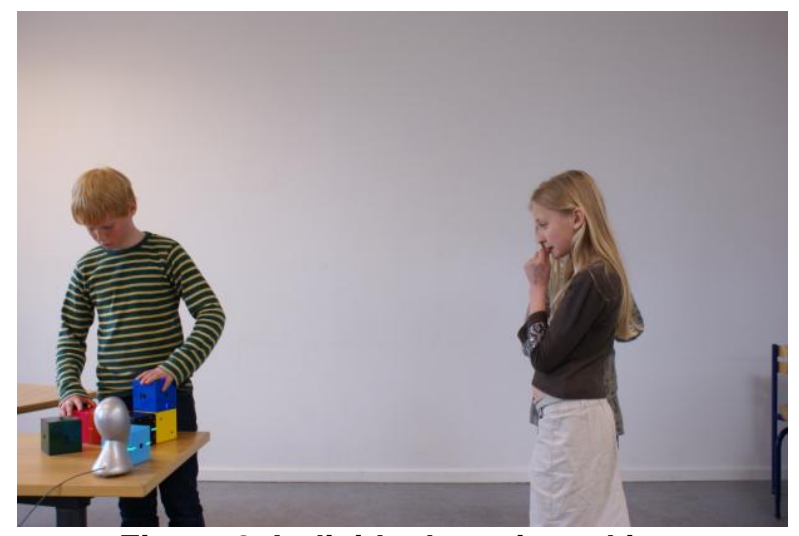

Figure 8. Individual music-making.

Some participants would start off by creating a combination of instruments and loops that they found fit together and subsequently change the entire structure, serving as another development of the song or tune. This resulted in a series of combinations of loops compromising not just a single mix but several developments of a tune. Also, when experimenting with the 'rock' tune, one participant would create an arrangement consisting of several different variations of loops and switching between these by moving the structure of the blocks around, while the other participants took turns to "play" the guitar solo, making up a jam session with the I-BLOCKS.

A striking observation made during these experiments was that every participating child was able to create their own musical piece within the short time of a session (lasting approximately 15 minutes). Everybody participated in collaborative as well as individual music-making and completed the task of creating a mix of their own, regardless of prior experience with instrumental music tuition

\section{Music-making with new technology}

Traditionally, being able to express oneself musically and engage in musical activities involving the production of sound, is somewhat determined by one's ability to play an actual instrument. In order to produce sound with an instrument one must possess a minimum of skills in regard to the actual handling of the instrument. In order to compose or in other ways carry out musical ideas a certain degree of craftsmanship and experience is needed [11][12].

As technological development and research generates different possibilities in approaching musicmaking and musical experimentation, new paradigms of musical expression, comprehension and creativity are formed. While technology plays an important role among musicians, for example in regard to recording 
music or performing music utilizing different technological tools, the development of sequencer programmes (Steinerg Cubase, Apple Logic etc.) as well as different types of midi controllers (e.g. midi keyboards, electronic drum kits, Korg's Kaossilator and new interfaces such as Zoundz, The Samchillian, Beat Bugs etc.) has had an important impact in regard to musical play among children and in music teaching in general. In the following we wish to point out some of the possible advantages linked to the use of new music technology, both in music education and out of school.

Norwegian professor of musicology and music education, Petter Dyndahl, has emphasized how "new" technology, for instance sequencing programmes or the midi standard as such, has made new ways of approaching musical creativity possible. According to Dyndahl a digital representation of music on a computer allows us to "mould directly" in the authentic, timbral substance [13], which brings to mind conceptions of music as a physical, mouldable object. Like modelling-wax - flexible and manageable in the hands of the user.

Sequencing programmes, for instance, allow us this kind of flexibility. We can record music of our own, sample existing recordings and manipulate sounds in an infinite amount of ways. Utilizing technology in the creation of music, we may experience a shortening of the distance between an idea and its actual aural representation. In fact, technology may even allow the non-musician access to a creative process involving music in ways previously unimaginable. Of particular interest in regard to musical expression and comprehension is the notion that by utilizing technology, such as musical toys or a computer, we may be able to express ourselves through music, and by doing so reveal skills of aesthetic sensitivity, originality or tonal imagery otherwise hidden as a result of lack of craftsmanship (Webster 1987). The music teacher may realize that pupils who have troubles developing instrumental skills or may lack interest in taking up an instrument, actually possess skills in regard to knowledge of musical genres, having a well-developed sense of aesthetics in music or being very original in their musical expression when utilizing "untraditional" tools in their creation of music.

While projects such as the aforementioned Beat Bugs or the Audio Cubes allow the user to create rhythmic patterns or experiment with harmonic and timbral sound material, the RoboMusicKids project aim to allow children to experiment and manipulate with pre-recorded musical sequences or elements, reminiscent of the way a DJ remixes existing tracks.
The I-BLOCKS serve as a multi-track mixer giving the user complete control over the progression and overall mix and arrangement of the track. Furthermore, IBLOCKS allow the user to isolate the separate elements of a musical piece, making it possible for him or her to understand the sound and musical role of different instruments, both in regard to musical arrangement and mix and in regard to music history, traditions of musical genres and sub-genres, technological possibilities in connection to studio equipment and the recording of music and so forth.

\section{Conclusion}

I-BLOCKS make it possible for the user to rearrange and manipulate musical elements and create an original musical output. In our experiments we found that the participants were aware of how they wanted their finished musical piece to sound, and were very selective in the process of "building" this. While music technology in some cases holds the risk of allowing us to simply generate a musical product and bypass the actual creative process, our experiments showed that even when utilizing a basic setup of six variations on six different instruments, the participants to a great extent were still able to express themselves creatively. This is not to say that the cubes allow users to make music as if they were composers, but the project does, in our opinion, show a possible direction for future development of musical teaching and learning.

Utilizing music technology in music education and out of school, may give children, or adults, the opportunity to be musically creative and create understanding of musical phenomena and structures. IBLOCKS represent an intuitive approach to music, both in regard to musical expression as well as musical comprehension and understanding. In the hands of the user, the I-BLOCKS turn music into a mouldable element due to their modular nature, allowing the user to explore its possibilities in a creative and playful way and serve as an intelligent tool that is manageable and flexible in regard to its user's approach to creativity and learning. Also, the modular nature of the IBLOCKS ensures that this system is easily expandable and our hope is that this will in turn enable us to provide more flexibility and freedom for the user in his or her experimentation with the music.

\section{References}


[1] J. Nielsen, and H. H. Lund, "Modular robotics as a tool for education and entertainment," Computers in Human Behavior, 24, 2008, Elsevier, pp. 234-248.

[2] J. Nielsen, and H. H. Lund, "Spiking neural building block robot with Hebbian learning," Intelligent Robots and Systems (IROS), 2, 2003, IEEE/RSJ,

[3] URL: http://www.apple.com/ilife/garageband/

[4] URL: http://www.ableton.com/

[5] URL: http://www.propellerheads.se/products/reason/

[6] G. Weinberg, R. Aimi, and K. Jennings, "The Beatbug network: a rhythmic system for interdependent group collaboration," Proceedings of the 2002 conference on New interfaces for Musical Expression, 2002, New Interfaces For Musical Expression, pp. 1-6.

[7] URL: http://www.sonycsl.co.jp/IL/projects/blockjam/

[8] B. Schiettecatte, and J. Vanderdonckt, "AudioCubes: a distributed cube tangible interface based on interaction range for sound design," Proceedings of the 2nd international conference on 2 nd international Conference on Tangible and Embedded interaction, 2008, ACM, New York, pp. 3-10.

[9] H. H. Lund, and M. Ottesen, "RoboMusic: a behaviorbased approach," In Artificial Life and Robotics Journal, 12, 2008, pp. 18-23.

[10] C. Quintana et. al. Learner-Centered Design. Reflections on the Past and Directions for the Future. In Sawyer, R.K. The Cambridge Handbook of the Learning Sciences. Cambridge University Press, 2006.

[11] G. Folkestad, "Computer based creative music making: Young people's music in the digital age", Göteborg: Acta Universitatis Gothoburgensis, 1996.

[12] P.R. Webster "Conceptual Bases for Creative Thinking in Music" In J. C. Peery, I. W. Peery \& T. W. Draper. Music and Child Development. New York: Springer-Verlag, 1987.

[13] Dyndahl, P., Music/technology/education. On digitised music education, its discourse and (self-)irony. (In Norwegian) Oslo: University of Oslo, 2002. 